\title{
Effects of surface charge density and distribution on the nanochannel electro-osmotic flow
}

\section{Bohumir Jelinek}

Center for Advanced Vehicular Systems, Mississippi State University, 200 Research Boulevard, Starkville, Mississippi 39759

E-mail: bj48@msstate.edu

Fax: 662-325-5433

\section{Sergio Felicelli*}

Mechanical Engineering Dept., Mississippi State University,

Mail Stop 9552, 210 Carpenter Building,

Mississippi State, Mississippi 39762

E-mail: felicelli@me.msstate.edu

*Corresponding author

\section{Paul F. Mlakar and John F. Peters}

U.S. Army ERDC, 3909 Halls Ferry Rd, Vicksburg, Mississippi 39180

E-mail: Paul.F.Mlakar@erdc.usace.army.mil E-mail: John.F.Peters@erdc.usace.army.mil

\begin{abstract}
Surface charge density and distribution dependence of a nanochannel electro-osmotic flow was examined using a molecular dynamics (MD) model. Systems consisting of $\mathrm{Na}^{+}$and $\mathrm{Cl}^{-}$ions in water confined between crystalline walls with varying negative charge on inner surfaces in an external electric field were investigated. At low surface charge densities, water flows as expected by common interpretations of electro-osmosis. At intermediate surface charge density, the flow exhibits a maximum. Strongly charged surfaces cause adsorption of counterions, immobilization of the near-wall fluid layers, and subsequent flow reversal. An effect of increase in the viscosity of water near the strongly charged surface was demonstrated. When the discrete $-1 e$ charge was distributed on a subgrid of surface atoms, the flow deteriorated and reversed at much lower surface charge densities than when all the surface atoms carried equal partial charge.
\end{abstract}

Keywords: nanochannel; electrokinetic flow; electro-osmosis; flow reversal; surface charge; charge density; charge distribution; density effects; viscosity; MD; molecular dynamics.

Copyright (c) 2009 Inderscience Enterprises Ltd. 
Reference to this paper should be made as follows: Jelinek, B., Felicelli, S. D., Mlakar, P. F., and Peters, J. F. (2011) 'Effects of surface charge density and distribution on the nanochannel electro-osmotic flow', Int. J. of Theoretical and Applied Multiscale Mechanics, Vol. 2, No. 2, pp.165-183.

Biographical notes: Bohumir Jelinek received his $\mathrm{PhD}$ in Physics from Mississippi State University (MSU) with a minor in Mechanical Engineering in 2008. He is a post-doctoral research associate at the Center for Advanced Vehicular Systems, MSU. His research interests include molecular dynamics and density functional theory simulations applied to electrokinetic phenomena and alloy design.

Sergio Felicelli received his $\mathrm{PhD}$ in Mechanical Engineering from University of Arizona in 1991. He is a Professor in the Mechanical Engineering Department, MSU. His research involves computational modeling of solidification phenomena, with application relevant to the aerospace and automotive industries.

Paul F. Mlakar is a Senior Research Scientist at the U.S. Army Engineer Research and Development Center (ERDC) in Vicksburg, Mississippi. He received his $\mathrm{PhD}$ in Engineering Science from Purdue University in 1975. He is a registered professional engineer in the state of Mississippi, the author of 150 technical publications, distinguished member of the American Society of Civil Engineers, and a fellow of the American Concrete Institute and the Society of American Military Engineers.

John F. Peters is a Senior Research Scientist at the U.S. Army Engineer Research and Development Center (ERDC) in Vicksburg, Mississippi. He received his $\mathrm{PhD}$ in Civil Engineering from the University of Illinois, Chicago in 1983. His research is in the area of modeling concrete at nanoscale, finite-element and discrete element modeling of near-surface phenomena in soils.

\section{Introduction}

Electro-osmosis is a movement of liquid in porous media under the influence of an applied electric field. When in contact with liquid, the pore surface acquires an electric charge and attracts counterions from the solution. The counterions near the pore surface form a layer that drives the electro-osmotic flow. Since dimensions of the counterion layer can be comparable to intermolecular distances, molecular dynamics (MD) models provide suitable tools to study electro-osmosis. Electroosmotic flow of water with $\mathrm{Cl}^{-}$ions (Freund 2002) and of a model electrolyte with $\mathrm{NaCl}$ (Zhu et al. 2005) in a simplified channel, of water with $\mathrm{NaCl}$ in a silicon channel (Qiao \& Aluru 2004), and of $\mathrm{NaCl} / \mathrm{NaI}$ in a model hydrophobic and hydrophilic channels (Huang et al. 2007) were subject of past studies. Electroosmotic flow of water with $\mathrm{KCl}$ (Joseph \& Aluru 2006) and $\mathrm{NaCl}$ (Lorenz et al. 
2008), and of multi-ionic solutions in clays (Rotenberg et al. 2007) were also explored by means of MD simulations. Moreover, MD techniques improve understanding of transport processes in biology (Han et al. 2006, Cory et al. 2007, Gumbart et al. 2009, Cruz-Chu et al. 2009, Lin et al. 2010).

The present study examines factors affecting the direction and intensity of an electro-osmotic flow in a slab nanochannel. The system of Qiao \& Aluru (2004) was chosen as a reference because it exhibits a flow reversal; that is, it predicts water flow in the direction opposite to common interpretations of electro-osmosis. MD simulations of $\mathrm{CaCl}_{2}$ in realistic silica channels by Lorenz et al. (2008) are another interesting case of predicting electro-osmotic flow in anomalous direction. To check the robustness of these predictions and to identify main factors causing the flow reversal, parameters of the reference system were systematically altered, including varying thermostating techniques and modifying the surface structure. Effects of surface charge density, shown experimentally by Schoch et al. (2005), its distribution, and ionic strength of a solution were investigated. Variation of water viscosity near strongly charged surface was estimated and its effects on the velocity profile of water were demonstrated. The observations lead to conclusion that surface charge distribution and density played the most important roles in whether flow reversal occurred.

\section{MD potentials}

Models based on classical Lennard-Jones (LJ) force fields and Coulomb electrostatic interactions with Simple Point Charge Extended (SPC/E) (Berendsen et al. 1987) model were used to represent the interactions between ions, water molecules, and channel wall atoms. LJ contribution of atoms $i$ and $j$ to the total potential energy is

$$
V_{L J}(r)=4 \epsilon_{i j}\left[\left(\frac{\sigma_{i j}}{r}\right)^{12}-\left(\frac{\sigma_{i j}}{r}\right)^{6}\right]
$$

where $\epsilon_{i j}$ is the depth of the potential well and $\sqrt[6]{2} \sigma_{i j}$ is the minimum energy distance, each depending on the species of the $i^{t h}$ and $j^{t h}$ atoms (Table 1). LJ parameters were taken from the GROMACS package (Hess et al. 2008). According to Patra \& Karttunen (2004), these perform similarly to other potentials of $\mathrm{NaCl}$ in aqueous solution. They also result in the flow reversal as observed in the reference system by Qiao \& Aluru (2004). Application of wall-fluid LJ parameteres from Huang et al. (2007) leads to similar results (Figure 7), without significant impact on the qualitative conclusions of present work. The Coulomb electrostatic potential energy contribution is

$$
V_{C}(r)=\frac{1}{4 \pi \varepsilon_{0}} \frac{q_{i} q_{j}}{r}
$$

where $\varepsilon_{0}$ is vacuum permittivity, $q_{i}$ and $q_{j}$ are charges of $i$ th and $j$ th atoms, and $r$ is the distance between $i$ th and $j$ th atoms. The Particle-Particle Particle-Mesh (PPPM) (Hockney \& Eastwood 1989, Plimpton et al. 1997) method was used for long range electrostatics, with interactions further than $11 \mathrm{~A}$ computed in reciprocal 
space. The LJ interactions were cut to zero at the same distance, which should be satisfactory to avoid spurious effects of short cutoff distance (Bonthuis et al. 2009).

Table 1 Parameters of Lennard-Jones potentials. Value of $\sigma_{i j}$ is in $\AA, \epsilon_{i j}$ in cal $/ \mathrm{mol}$.

\begin{tabular}{c|ccccccccc} 
ij & OO & OSi & ONa & OCl & $\mathrm{SiNa}$ & $\mathrm{SiCl}$ & $\mathrm{NaNa}$ & $\mathrm{NaCl}$ & $\mathrm{ClCl}$ \\
\hline$\sigma_{i j}$ & 3.17 & 3.27 & 2.86 & 3.75 & 2.95 & 3.88 & 2.58 & 3.38 & 4.45 \\
$\epsilon_{i j}$ & 155 & 301 & 47.9 & 129 & 92.9 & 249 & 14.8 & 39.6 & 106
\end{tabular}

\section{Simulation setup}

The authors first reproduced the reference system of Qiao \& Aluru (2004), the summary of which is given next. The dimensions of the solution region were $4.66 \times 4.22 \times 3.49 \mathrm{~nm}$. Channel walls perpendicular to the $z$ axis were formed by four [111] oriented layers of $\mathrm{Si}$ atoms in a diamond crystal structure, each wall being $0.39 \mathrm{~nm}$ thick. Periodic boundary conditions were applied in the $x$ and $y$ directions. The size of the simulation cell in the $z$ direction was extended to three times outermost-to-outermost wall layer distance to mitigate electrostatic interactions of periodic images in the $z$ direction (Yeh \& Berkowitz 1999). The electric field of $0.55 \mathrm{~V} / \mathrm{nm}$ was applied in the positive $x$ direction. Each channel wall consisted of 616 fixed $\mathrm{Si}$ atoms. A charge of -35 e was distributed uniformly on all 154 atoms of the innermost surface layer of each wall, introducing partial charge of $-0.227 \mathrm{e} /$ atom. That corresponds to a mean surface charge density $\Sigma=-0.285 \mathrm{C} \mathrm{m}^{-2}$, which is close to the $\Sigma=0.2 \mathrm{C} \mathrm{m}^{-2}$ for a fully ionized surface (Israelachvili 1985) or to the $\Sigma=-0.1 \mathrm{C} \mathrm{m}^{-2}$ measured at silica surface (Dove \& Craven 2005). The solution was formed of $108 \mathrm{Na}^{+}, 38 \mathrm{Cl}^{-}$ions, and 2144 water molecules confined between the negatively charged channel walls. Thus the whole system was electrically neutral with a resulting $\mathrm{NaCl}$ concentration in the channel center of $\approx 1.2 \mathrm{M}$. Final configuration, excluding water molecules, is shown in Figure 1. Water molecules and ions were inserted between the walls using the PACKMOL package (Martínez et al. 2009), followed by the energy minimization of atomic positions using the conjugate gradient method. Since the numbers of ions were changing, the distance between the channel walls was adjusted by applying external pressure of $10 \mathrm{~atm}$ on one wall and fixing the other wall for up to $1 \mathrm{~ns}$. After pressure adjustment the positions of both walls were fixed, after which the system was equilibrated by 2 ns of MD simulation without an electric field. A timestep of $2 \times 10^{-15} \mathrm{~s}$ was used for the leapfrog integration (Rapaport 2004) of Newton's equations of motion. Finally, a 22 ns MD run was performed with external electric field. Bonds of water molecules were constrained by the SHAKE algorithm (Ryckaert et al. 1977). The solution temperature of $300 \mathrm{~K}$ was controlled by the Nosé-Hoover (Nosé 1984, Hoover 1985) selective thermostat, adjusting only $y$ and $z$ components of velocity to avoid alteration of the velocity component in the field direction. Velocity averages of water from the full ( $x, y$, and $z$ components) thermostat were mostly within error bars of the selective thermostat (Figure 2), thus having minimal effect on the flow reversal. Velocity profiles were also generated with profile-unbiased thermostat (PUT), which preserves the velocity profile across 
Figure 1 Simulation box without water molecules. Si wall atoms are gray, $\mathrm{Na}^{+}$ions red, and $\mathrm{Cl}^{-}$ions green. Water molecules are not shown. Electric field is applied in the positive $x$ direction.

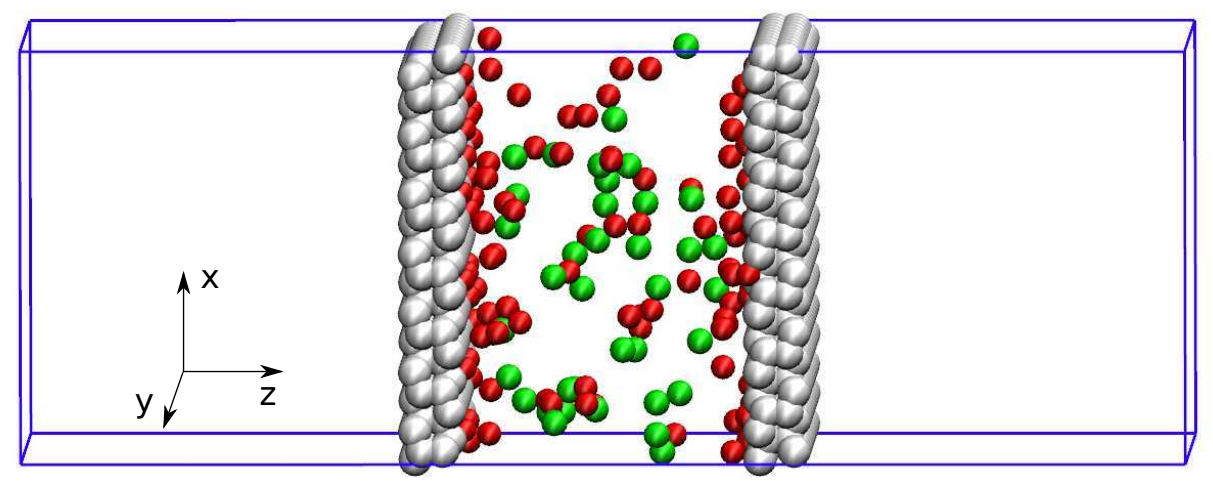

Figure 2 Comparison of water velocity profiles using full thermostat (adjusting $x, y$, and $z$ velocity components) and selective thermostat (adjusting $y$ and $z$ components only) for the reference system. Velocity samples are averaged over $\approx 0.026 \mathrm{~nm}$ wide bins parallel to the $x y$ plane that are then symmetrically averaged about the channel center. Averages, shown with error bars on only one side to avoid overlap, were obtained from ten simulations with different initial seeds of random Gaussian velocity distribution.

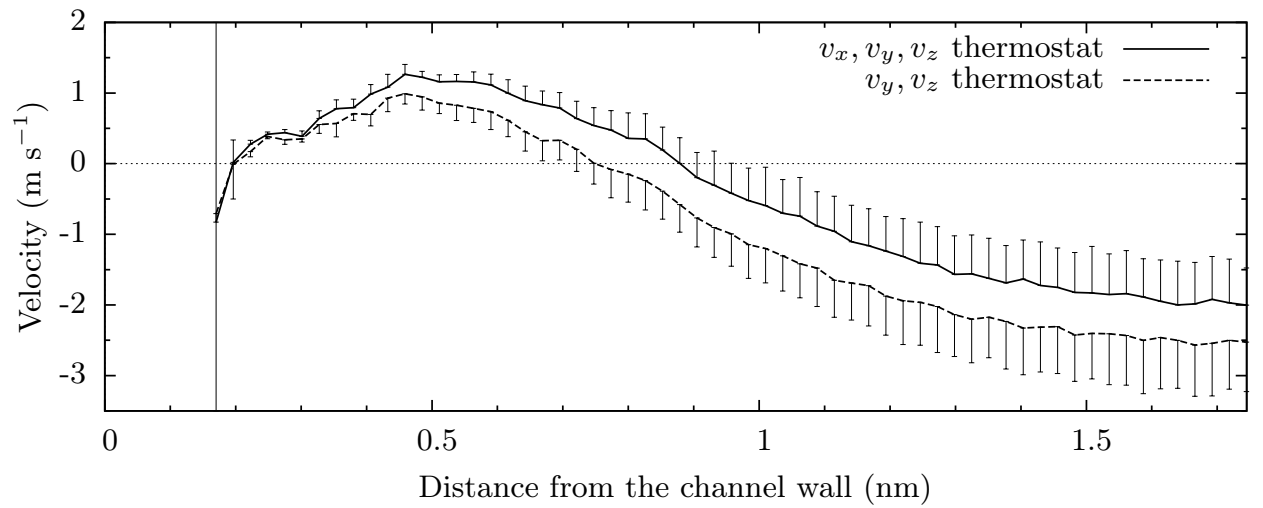

the channel (Evans \& Morriss 1986). PUT averages did not differ significantly from those obtained by selective thermostat, therefore the selective thermostat was applied.

To investigate main factors affecting the flow, predictions for flow reversal were checked in the reference system with each of the following modifications:

- The walls were extended to twice of the original thickness.

- Periodic boundary conditions were imposed in all three directions. 
- The field strength was lowered to $0.06 \mathrm{~V} / \mathrm{nm}$.

- Wall atoms were fixed on springs and thermostated separately.

- Crystal structure of Si walls was changed from the diamond [111] to fcc [111] with identical innermost surface layer.

- Original surface charge density of $-0.285 \mathrm{C} \mathrm{m}^{-2}$ and the number of ions were reduced.

- A discrete charge of -1 e was distributed on selected atoms of the innermost surfaces instead of uniformly charging all of them.

Of the above modifications, only the reduction of surface charge density suppressed the flow reversal. It was also found that the distribution of -1 e charge on surface atoms encouraged the flow reversal. Therefore, the effects of surface charge density and distribution were investigated in detail.

An issue encountered with the discrete -1 e charge was that if charged surface atoms were chosen randomly and two of them happened to be neighbors, then $\mathrm{Na}^{+}$ions penetrated the surface between the two, resulting in reduction of the effective surface charge density. To avoid the penetration of ions into surface layer, the discrete -1 e charge was placed randomly only on the atoms of a regular hexagonal subgrid of the surface layer, ensuring that no two neighboring surface atoms carried a charge. To make the subgrid periodic, surface dimensions were increased to $5.32 \times 4.61 \mathrm{~nm}$. Therefore, the surface area of systems with discrete -1 e surface charges was larger than the surface area of systems with all surface atoms carrying uniform partial charge and the number of water molecules was proportionally increased to 2673 .

\section{Concentration profiles}

Layering of fluid particles near a flat surface is characteristic for charged (Xia) \& Berkowitz 1995, Lyklema et al. 1998, Netz 2003) and also for uncharged surfaces (Abraham 1978). Cross-channel concentration profiles of individual species calculated here for the uniform partial surface charge at two representative surface charge densities and for the discrete -1 e surface charge are shown in Figure 3. Results for the reference system, agreeing well with those reported by Qiao \& Aluru (2004), manifest formation of alternatively charged layers of atoms parallel to channel walls. Negatively charged Si wall surface attracts both positively charged $\mathrm{Na}^{+}$ions and $\mathrm{H}$ atoms (carrying a partial positive charge of $+0.4238 \mathrm{e} /$ atom for $\mathrm{SPC} / \mathrm{E}$ water) from $\mathrm{H}_{2} \mathrm{O}$ molecules, forming the first near-wall concentration peak. An adjacent layer is formed of $\mathrm{O}$ atoms (carrying a partial negative charge of -0.8476 e/atom for SPC/E water) from $\mathrm{H}_{2} \mathrm{O}$ molecules. Five noticeable layers of alternating charge density (see also bottom plot in Figure 9 ) are formed - four ionic layers can be seen in Figure 5 and the fifth is a negatively charged layer of $\mathrm{O}$ atoms $0.25 \mathrm{~nm}$ from the channel wall (Figure 3). Interestingly, at the less strong surface charge density of $\Sigma_{p}=-0.143 \mathrm{C} \mathrm{m}^{-2}$, the first near-wall high concentration peak of $\mathrm{Na}^{+}$ions (located $0.14 \mathrm{~nm}$ from the channel center) disappears, and the second peak $(0.38 \mathrm{~nm}$ from the wall) flattens out, leading to an increase in 
Figure 3 Concentrations of $\mathrm{H}$ and $\mathrm{O}$ atoms from $\mathrm{H}_{2} \mathrm{O}$ molecules, $\mathrm{Na}^{+}$ions, and $\mathrm{Cl}^{-}$ ions symmetrically averaged across half of the channel with uniform partial surface charge of $\Sigma_{p}=-0.285 \mathrm{C} \mathrm{m}^{-2}$ (reference system), $\Sigma_{p}=-0.143 \mathrm{C} \mathrm{m}^{-2}$, and the discrete -1 e surface charge of $\Sigma_{1 e}=-0.144 \mathrm{C} \mathrm{m}^{-2}$. The inset plot is a magnified portion of a larger plot.
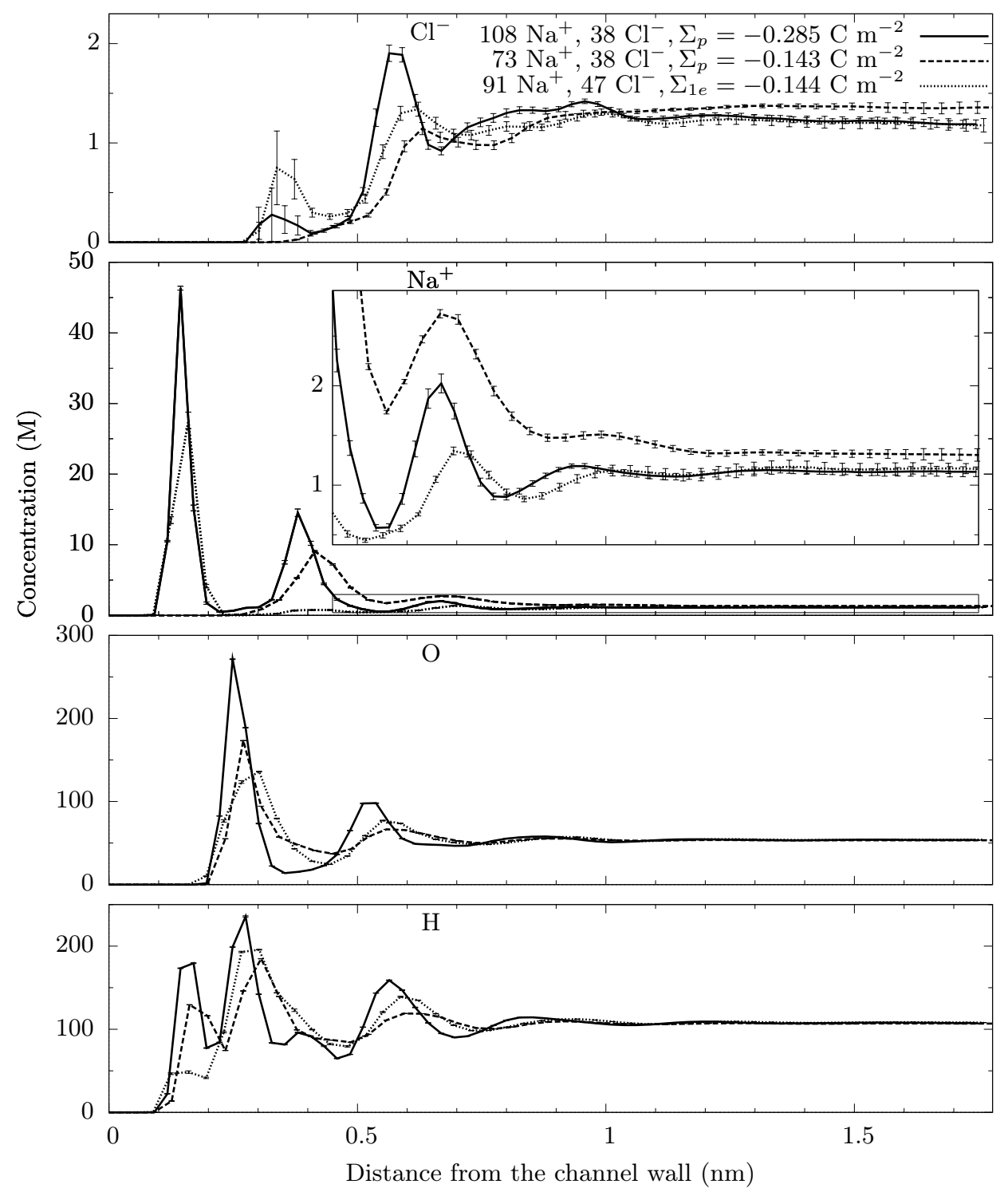

concentration of $\mathrm{Na}^{+}$ions towards the channel center. At the discrete surface charge of $\Sigma_{1 e}=-0.144 \mathrm{C} \mathrm{m}^{-2}$, the first (adsorption) peak in $\mathrm{Na}^{+}$concentration is present, implying larger "adsorption capacity" of the discretely charged surface than uniformly partially charged surface with similar charge density. The second 
Figure 4 Potential energy profile of a single Na ion in an empty channel along the line passing through its adsorption site. Upper curves are from PPPM with complete walls of the reference system, showing LJ energy $\left(\mathrm{V}_{L J}\right)$ and short range component of Coulomb energy $\left(\mathrm{V}_{C S}\right)$ of $\mathrm{Na}^{+70}$. Lower curves are energies of three neighboring surface atoms and $\mathrm{Na}^{+}$ion from equations 11 and $(2)$.

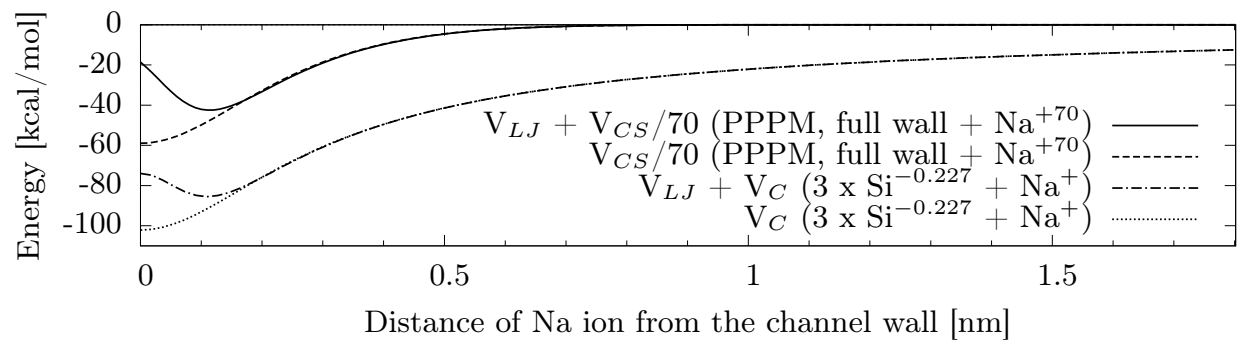

peak of $\mathrm{Na}^{+}$, on the contrary, is only marginally low. Moreover, orientational polarization of water molecules close to the channel wall can be deduced from the concentration profiles of $\mathrm{H}$ and $\mathrm{O}$ atoms in Figure 3 Because the first concentration peak of $\mathrm{H}$ atoms is closer to the wall than the first peak of $\mathrm{O}$ atoms, the near-wall water molecules reorient so that $\mathrm{H}$ atoms point to the surface. Visualization of MD trajectories shows that the first near-wall peak in the $\mathrm{Na}^{+}$concentration comes from the ions adsorbed on the channel surface, with the adsorption site located centrally above three neighboring innermost-surface atoms. Adsorption sites were also identified from the potential energy profile of a reference system with only wall atoms and a single $\mathrm{Na}$ ion positioned along the line parallel to the channel passing through the adsorption site. Two upper potential energy profiles in Figure 4 were obtained by the PPPM method for a system with complete walls of the reference configuration, showing only short range Coulomb energy component. Na ion had a charge of +70 e to keep the PPPM system electrically neutral, and the resulting Coulomb energy was divided by 70. Two lower potential energy profiles in Figure 4 were obtained from equations (1) and (2) for a system with only three innermostsurface atoms closest to the adsorption site and one $\mathrm{Na}^{+}$ion. The position of the potential energy minimum corresponds to the position of the first near-wall $\mathrm{Na}^{+}$ concentration peak (Figure 3). The potential energy minimum deepens and the adsorption intensifies for highly charged surface atoms. On the contrary, at low surface charge densities with all surface atoms carrying the same partial charge, ions don't adsorb on the surface at all.

\section{Driving force}

Since the net charge of a standalone water molecule is zero, its center of mass will not move in the presence of external electric field. The driving force for the electroosmotic flow comes from the electric field causing the movement of a fluid region with non-zero net charge. Each infinitesimally small volume of fluid with positive net charge will drive the flow in the direction of an external electric field, while the volume with negative net charge will drive the flow in the direction opposite to an 
external electric field. In the present system, the charge carriers are $\mathrm{Na}^{+}$and $\mathrm{Cl}^{-}$ ions, therefore the driving force per unit volume is defined as

$$
\mathbf{F}_{d}(z)=e\left[c_{\mathrm{Na}^{+}}(z)-c_{\mathrm{Cl}^{-}}(z)\right] \mathbf{E}_{e x t},
$$

where $e$ is the elementary charge, $c_{\mathrm{Na}^{+}}(z)$ and $c_{\mathrm{Cl}^{-}}(z)$ are ionic number densities across the channel (proportional to ionic concentrations-Figure 3), and $\mathbf{E}_{e x t}$ is an external electric field. Figure 5 shows cross-channel profiles of the driving force at two representative surface charge densities for the uniform partial surface charge, and at one surface charge density for the discrete -1 e surface charge. At the surface charge density of $\Sigma_{p}=-0.285 \mathrm{C} \mathrm{m}^{-2}$ (reference system), $\mathrm{Cl}^{-}$ions dominate $\mathrm{Na}^{+}$ions in two regions: one centered at $0.57 \mathrm{~nm}$ from the channel wall and the other one starting $0.73 \mathrm{~nm}$ from the channel wall that includes the channel center, thus introducing negative driving force. At the lower surface charge density of $\Sigma_{p}=-0.143 \mathrm{C} \mathrm{m}^{-2}$, the first near-wall peak in the driving force (from $\mathrm{Na}^{+}$ surface adsorption) is not present. $\mathrm{Cl}^{-}$ions do not significantly dominate $\mathrm{Na}^{+}$ions anywhere in the channel, and the driving force is mostly positive. The case with discrete surface charge density of $\Sigma_{1 e}=-0.144 \mathrm{C} \mathrm{m}^{-2}$ is similar to the case of $\Sigma_{p}=-0.285 \mathrm{C} \mathrm{m}^{-2}$, but with much lower second near-wall peak in the driving force and with regions of $\mathrm{Cl}^{-}$dominance shifted slightly towards the channel center. Near the channel center, concentrations of ions become more balanced, resulting in almost zero or marginally negative driving force.

The changes in ionic concentration profiles with surface charge density and directly related changes of the driving force are the primary mechanisms causing the negative water flow at high surface charge densities and positive water flow at low surface charge densities.

\section{Velocity profiles}

Figure 6 reveals a strong dependence of the flow on the surface charge density. At the uniform partial surface charge density of $\Sigma_{p}=-0.285 \mathrm{C} \mathrm{m}^{-2}$, flow reversal of water was observed - in agreement with the previous results (Qiao \& Aluru 2004). Water flow in the negative direction is caused by the negative driving force in a significant portion of the channel center, where $\mathrm{Cl}^{-}$ions dominate $\mathrm{Na}^{+}$ions (Figure 5). The first near-wall peak in $\mathrm{Na}^{+}$concentration does not affect the flow of water since these ions are adsorbed on the channel surface. The second peak also does not drive much water flow because of increased viscosity of water in the near-wall region at high surface charge density (Sec. 910 . At $\Sigma_{p}=-0.143 \mathrm{C} \mathrm{m}^{-2}$, water flows in the positive $x$ direction - as expected by common interpretations of electro-osmosis. The positive flow is caused by the prevalent positive driving force (Figure 55. Redistribution of $\mathrm{Na}^{+}$ions further from the channel wall at lower surface charge density stimulates the movement of water in the positive $x$ direction. Similar effect - when increased temperature causes charge redistribution and suppresses the flow reversal - was presented by Jelinek et al. (2009). Flow with a discrete -1 e surface charge distributed on a subgrid of surface atoms at the density of $\Sigma_{1 e}=-0.144 \mathrm{C} \mathrm{m}^{-2}$ is similar to the case of strongly uniformly charged surface.

Flow reversal at high surface charge density was attributed to (a) surface adsorption of $\mathrm{Na}^{+}$ions, and to (b) increase of the near-wall water viscosity at high 
Figure 5 Symmetrically averaged driving force for the fluid flow, equation (3). The inset plot magnifies values away from the channel wall-where the driving force is more negative at higher charge density per atom.

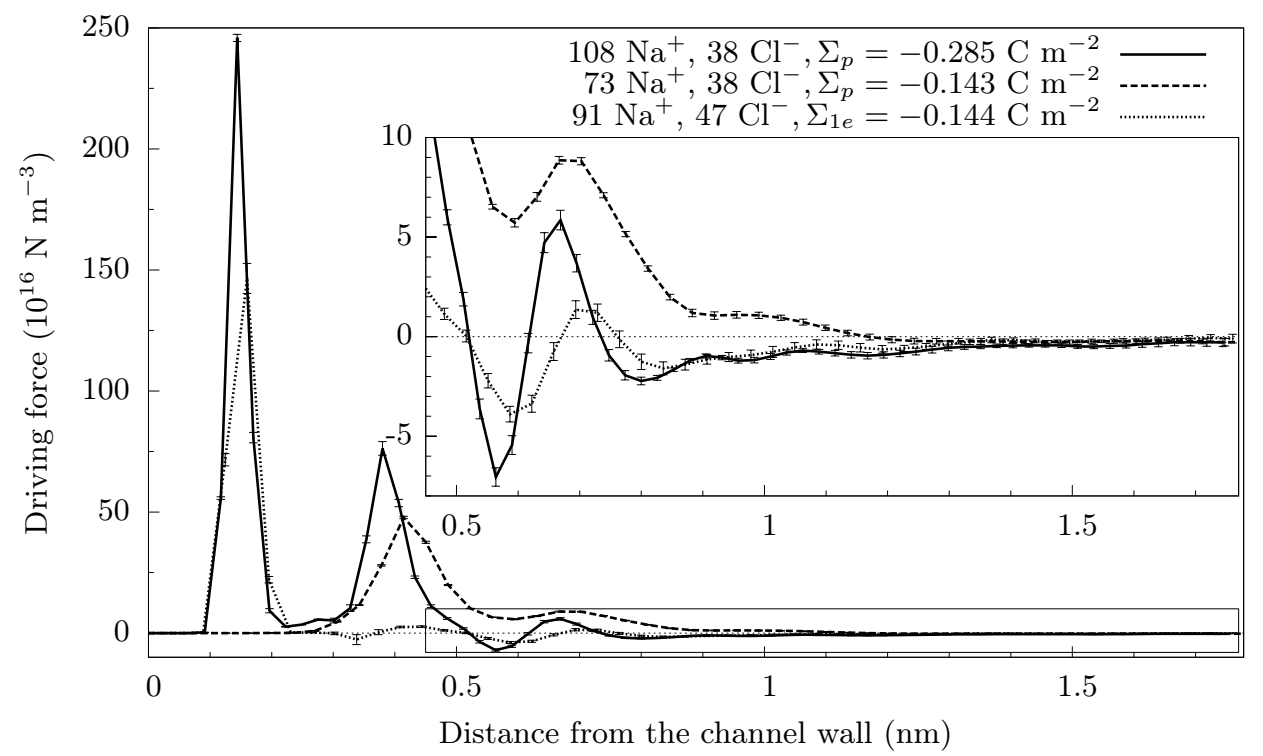

surface charge density (Sec. 9 10). Note that even though $\mathrm{Cl}^{-}$ions in water move faster than $\mathrm{Na}^{+}$ions (Figure 6), that does not affect water velocity profile. Water movement is not governed by the ionic velocities, but by the profile of the driving force. Velocity of ions relative to water is given by their respective mobilities $\mu_{i}$ according to the definition of mobility $\mathbf{v}_{\mathbf{i}}=\mu_{i} \mathbf{E}$. The LJ potential properly reflects the experimental mobility of $\mathrm{Cl}^{-}$ions being higher than the mobility of $\mathrm{Na}^{+}$ions.

\section{Zeta potentials}

Zeta potential defined by MD simulations is proportional to the water velocity in the channel center

$$
\zeta=\frac{u_{x}\left(z_{\text {center }}\right) \eta}{\varepsilon_{0} \varepsilon_{\mathrm{r}} \mathrm{E}_{x}}
$$

where $\eta=0.852 \times 10^{-3} \mathrm{~Pa}$ s is the water viscosity at $\mathrm{T}=300 \mathrm{~K}, \varepsilon_{0}$ is the permittivity of vacuum, $\varepsilon_{r}=68$ is the relative permittivity of water, and $\mathrm{E}_{x}$ is the strength of applied electric field.

Zeta potentials for the uniform partial charge distributed on all innermost surface atoms were evaluated for the channel with walls of diamond [111] and fcc [111] structures (Figure 7). The flow reversal (negative $\zeta$ potential) was observed at strong surface charge densities, resulting in small negative values of zeta potential. At less negative surface charge densities, water flows in the expected direction. The surface of fcc [111] structure generates stronger flow than the surface of 
Figure 6 MD velocity profiles of water molecules and ions with the partial surface charge of $\Sigma_{p}=-0.143 \mathrm{C} \mathrm{m}^{-2}$ (top), $-0.285 \mathrm{C} \mathrm{m}^{-2}$ (middle, note that Figure 9 shows details of water velocity for the reference system), and the discrete -1 e surface charge of $\Sigma_{1 e}=-0.144 \mathrm{C} \mathrm{m}^{-2}$ (bottom). Negative flow means movement in the direction opposite to applied electric field. Velocity labeled $\mathrm{H}_{2} \mathrm{O}$ is a velocity of oxygen atoms from $\mathrm{H}_{2} \mathrm{O}$ molecules. Error bars (for $\mathrm{Na}^{+}$and $\mathrm{H}_{2} \mathrm{O}$ they are represented by rectangles) were obtained by comparing averages of ten simulations with different seeds of initial Gaussian random velocity distribution. Large error bars near the channel wall are due to low concentrations (Figure 3).

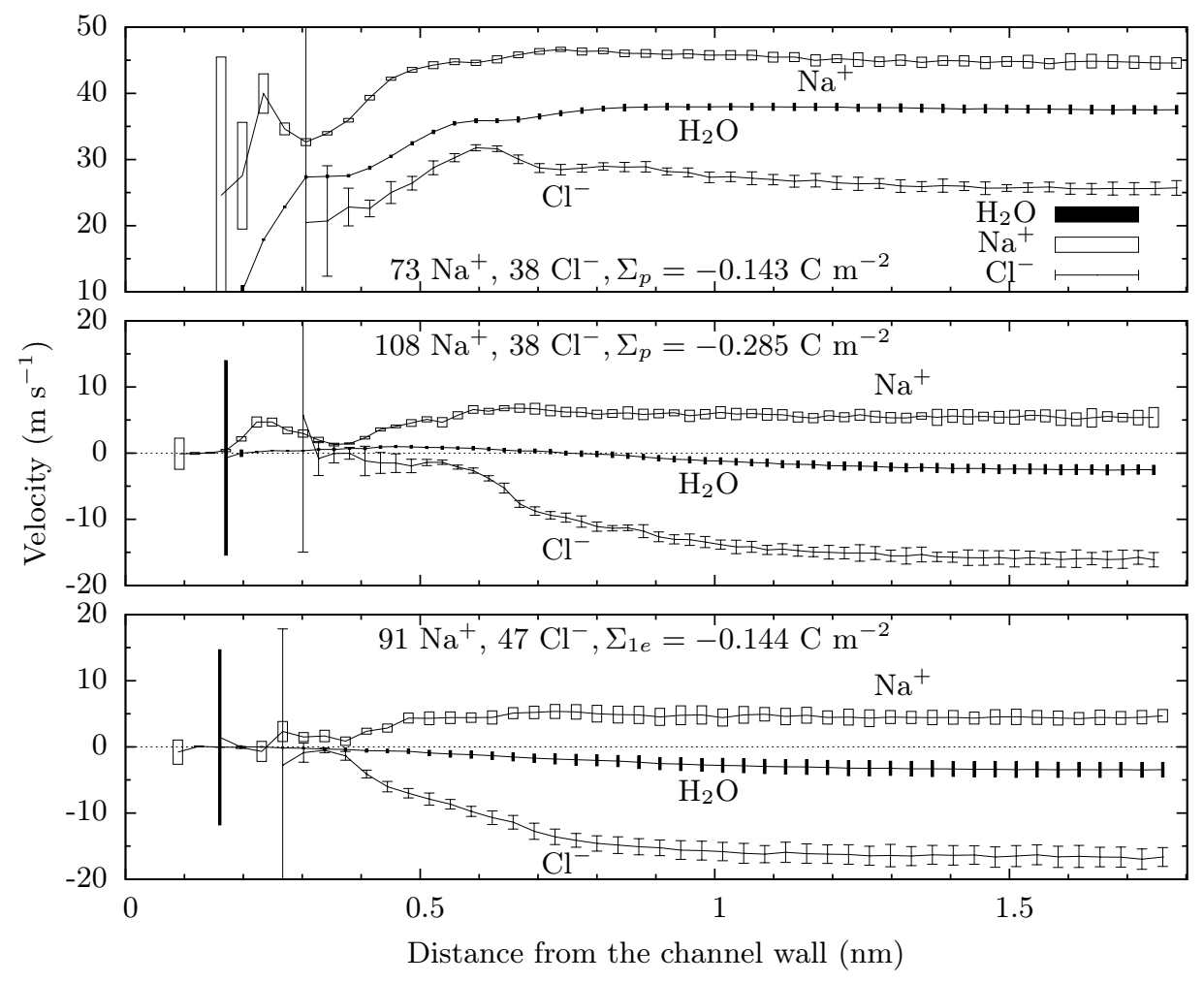

diamond [111] structure, with the largest zeta potential of $250 \mathrm{mV}$ at the surface charge density of $\Sigma_{p}=-0.073 \mathrm{C} \mathrm{m}^{-2}$. Similar value of $200 \mathrm{mV}$ with a flow weakening at increased surface charge density was reported by Huang et al. (2007) for the fcc [100] surface. The surface of diamond [111] structure generates less flow, with the largest zeta potential of $110 \mathrm{mV}$ at $\Sigma_{p}=-0.106 \mathrm{C} \mathrm{m}^{-2}$. When LJ parameters of the reference system were changed to those of the hydrophilic surface described in Huang et al. (2007), the zeta potential decreased by $12 \mathrm{mV}$, corresponding to stronger flow reversal (Figure 7). When ionic strength (number of ionic pairs) was decreased at the constant surface charge density (compare configurations 88:38 to 50:0 for fcc, and 108:38 to 78:8 for diamond wall structure in Figure 7), slightly more positive flow was observed - caused by a stronger dominance of positive ions across the whole channel. Since the lower number of ionic pairs 
leads to lower ionic concentration in the channel center, we can conclude that lower ionic concentration at constant surface charge density leads to increase in the flow. For the case of uniform partial charge distributed on all atoms of the innermost channel surface, flow reversal only appears at strong surface charge densities.

Zeta potentials for the case with discrete -1 e charge distributed on a subset of the innermost surface atoms of the diamond [111] and fcc [111] surface were also evaluated (Figure 8). For a case with the discrete +1 e charge, Freund (2002) reported about $30 \%$ higher midchannel water velocity than for uniform charge with a midchannel $\mathrm{Cl}^{-}$concentration of $0.01 \mathrm{M}$ and $\Sigma=-0.0817 \mathrm{C} \mathrm{m}^{-2}$. Present simulations, with negatively charged surface and two-ion solutions at higher ionic concentrations, show an opposite effect-zeta potentials with a discrete - 1 e surface charge are lower, even negative, and the flow reversal starts at lower surface charge densities (in absolute value) than in the case of uniform partial charge on all surface atoms. Early flow reversal for discretely charged surface is caused by adsorption of counterions on -1 e charged surface atoms. Figure 3 shows that for the discretely charged surface, the first near-wall peak of $\mathrm{Na}^{+}$ions (adsorption) is still present at $\Sigma_{1 e}=-0.144 \mathrm{C} \mathrm{m}^{-2}$. On the contrary, for uniformly charged surface, the mean surface charge density of $\Sigma_{p}=-0.143 \mathrm{C} \mathrm{m}^{-2}$ is not strong enough to adsorb

Figure 7 Dependence of zeta potential on the surface charge density for uniform partial charge distributed on all innermost surface atoms of Si walls in the diamond [111] and fcc [111] crystal structures. Labels designate number of $\mathrm{Na}^{+}$ : number of $\mathrm{Cl}^{-}$ions, number of water molecules was 2144 . Value marked with star was obtained with hydrophilic surface parameters of Huang et al. (2007).

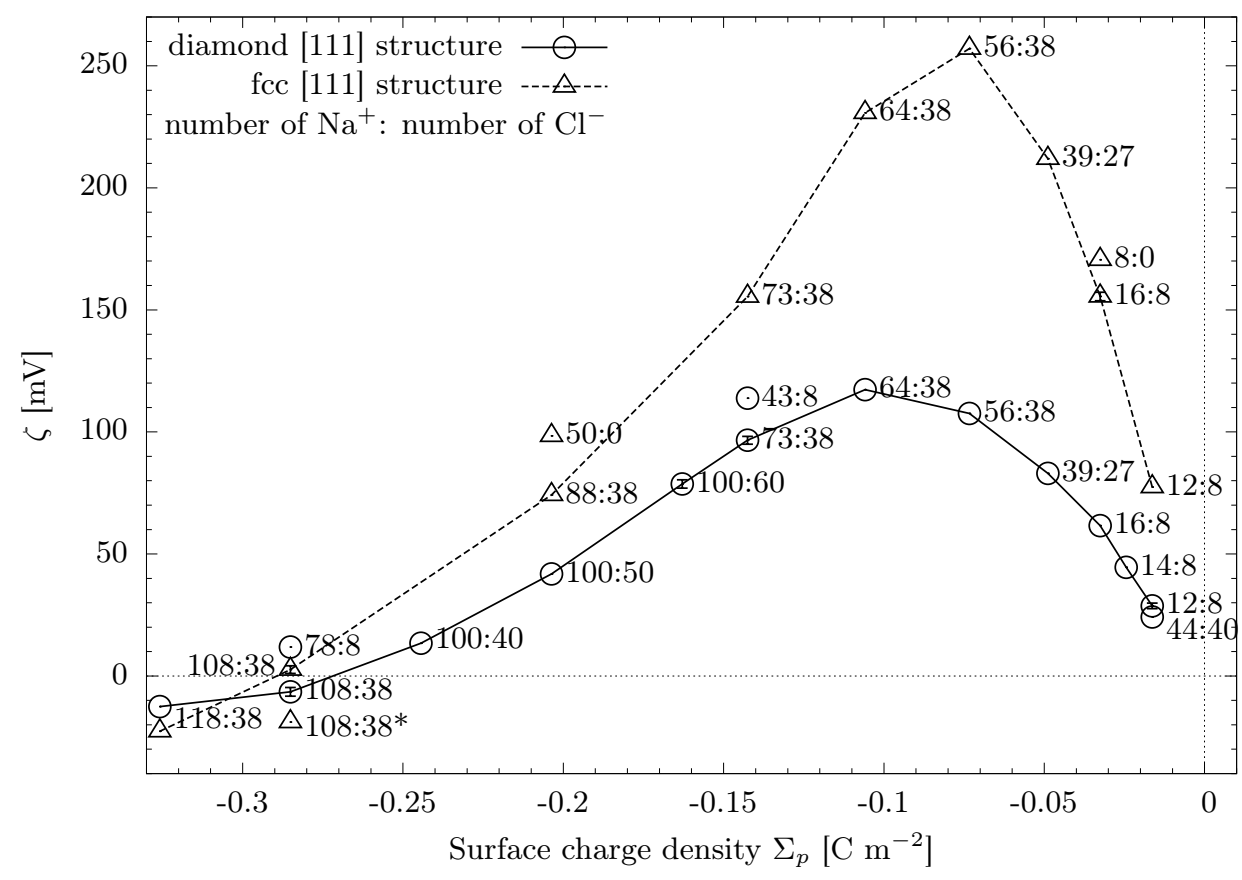


counterions and the $\mathrm{Na}^{+}$adsorption peak is not observed. Notice that if only $\mathrm{Na}^{+}$ ions are present in the solution (no $\mathrm{Cl}^{-}$ions), the flow reversal cannot appear.

\section{Prediction of velocity profiles from MD charge distribution}

The velocity profile of water for the reference system at $\Sigma_{p}=-0.285 \mathrm{C} \mathrm{m}^{-2}$ obtained as a bin-average of oxygen atom velocities from ten MD simulations is represented by circles with error bars in the middle plot of Figure 9. Furthermore, given ionic number densities $c_{\mathrm{Na}^{+}}(z), c_{\mathrm{Cl}^{-}}(z)$, and water viscosity $\eta(z)$, the velocity profile of water can be predicted using Stokes equation (Hunter 1981, 1987)

$$
\frac{d}{d z}\left[\eta(z) \frac{d u_{x}(z)}{d z}\right]=-F_{d}(z)
$$

with $\mathrm{d} u_{x}(z) / \mathrm{d} z=0$ at the channel center. Discussion of second boundary condition follows shortly. Magnitude of the driving force, $F_{d}(z)$, is given by equation (3).

Close to the channel center, constant viscosity can be assumed. Using $\eta_{300 \mathrm{~K}}=$ $0.852 \times 10^{-3}$ Pa s (ThermExcel 2003), the solutions $u_{x}(z)$ of equation (5) supplied with MD ionic charge densities (lower plot of Figure 9) are represented as dot-dash curves on the middle plot in Figure 9. Three different curves represent three different locations of non-slip boundary condition. The middle one matches MD velocity

Figure 8 Dependence of zeta potential on the surface charge density for the discrete -1 e charge distributed on a subgrid of the innermost surface atoms of Si walls in the diamond [111] and fcc [111] crystal structures. Labels designate number of $\mathrm{Na}^{+}$: number of $\mathrm{Cl}^{-}$ions, the number of water molecules was 2673 . With a discrete -1 e charge, the flow reversal starts at lower surface charge density than when all the surface atoms carry a uniform partial charge (Figure 7. notice that the scale of zeta potential axis is 4 times lower there).

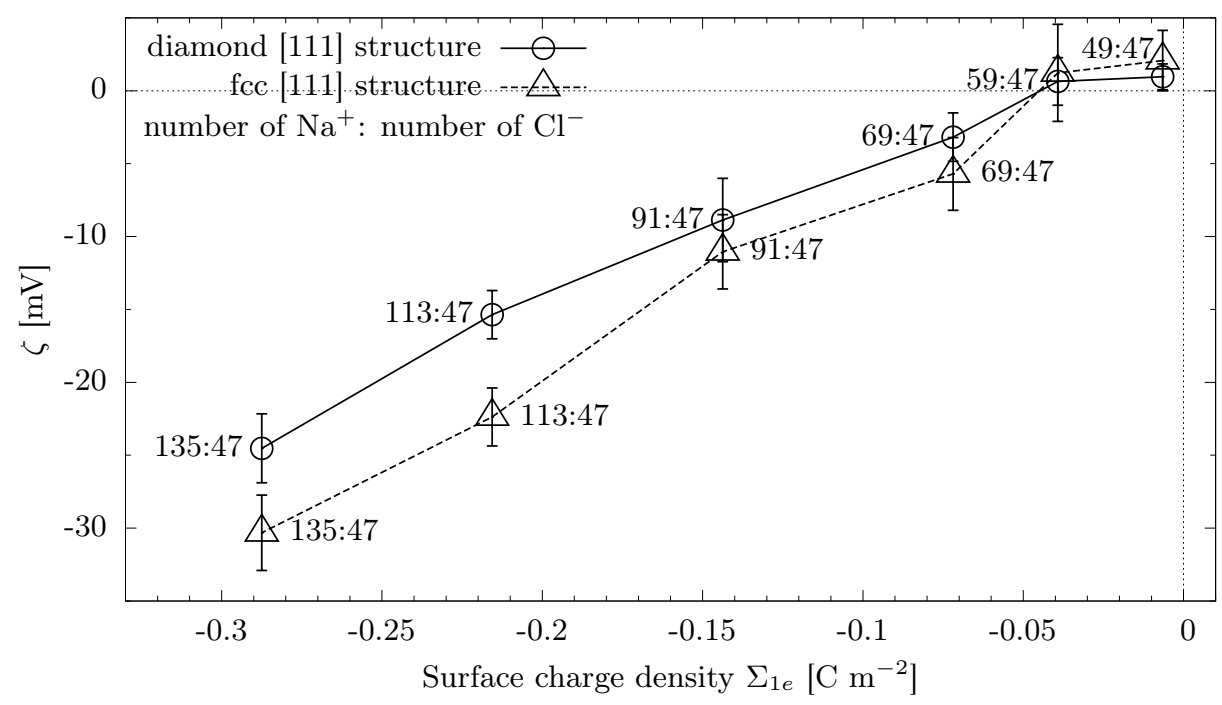


profiles in the channel center, but it seems to overestimate changes of the velocity close to the channel surface - where the environment is strongly inhomogeneous due to the high concentration of ions and charge layering of water molecules (lower plot in Figure 9p. Given that an increase in the viscosity of water was also found near an uncharged surface (Sendner et al. 2009), all suggest that the prediction of velocity profile could be improved by increasing viscosity close to the channel wall - as it will be demonstrated next.

\section{Estimation of water viscosity}

The cross-channel water viscosity profile for the reference system was estimated following the method of Davis \& Leighton (1987) as implemented by Freund (2002). The same method, without smoothing of velocity profile, was used by Qiao \& Aluru (2003). To simplify equations, the coordinate system with $\mathrm{z}=0$ at the channel center will be used. Integration of Stokes equation (5) (which is based on the definition of viscosity and can also serve as an estimator of $\eta(z)$ ) leads to viscosity estimate

$$
\left.\eta(z)\right|_{z=z_{0}}=\frac{-\int_{0}^{z_{0}} F_{d}(z) d z}{\left.\frac{d u_{x}(z)}{d z}\right|_{z=z_{0}}} .
$$

The estimate diverges near the points where the derivative of velocity is zero, as is appropriate, since the viscosity cannot be estimated using this method in the region with zero shear strain, for which the denominator is zero. The numerator term represents the shear stress (Davis \& Leighton 1987).

To obtain smooth derivative of $u_{x}(z)$, the velocity profile was approximated by the sum of harmonic components

$$
u_{x f i t}(z)=\sum_{n=0}^{7} a_{n} \cos \left(n \pi \frac{z}{h}\right)
$$

The $h$ is the distance of the furthest oxygen atom from the channel center. In contrast to the original approximation of Freund (2002), the exponential term is excluded. To exploit the symmetry of the system, the origin of cosine components is set to the channel center.

The velocity, its approximation, the viscosity profile estimated by equation (6), and the experimental viscosity of water (ThermExcel 2003) for the reference system are shown in Figure 9. Note that in a region close to the channel center, the estimated viscosity (line made of circle symbols on the top plot in Figure 9 at its minimum reproduces the experimental viscosity (solid horizontal line). At high surface charge densities (in absolute value), viscosity of the near-wall water layers increases significantly. Therefore the near-wall $\mathrm{Na}^{+}$ions will not drive much water flow. Conversely, at lower surface charge densities, the near-wall water will become less viscous and the changes in velocity gradient of water near the channel wall will increase. 
Figure 9 Bottom plot shows MD charge densities of ions (solid curve) and water (double-dot-dash curve) for the reference system. Middle plot shows MD velocity profile of water (circles with error bars), its fit to equation (7) (dotted curve), and water velocity profiles predicted by solving Stokes equation (5) with MD ionic charge distributions - assuming constant viscosity (dot-dash lines) and variable viscosity (solid lines). Three adjacent solutions apply non-slip BCs at three equidistant points. Top plot shows water viscosity estimate from equation (6) (circles), its fit to equation (8) (solid curve), experimental viscosity (horizontal dot-dash line) and $\mathrm{BC}$ location (solid vertical line). The inset plots are magnified portions of larger plots.

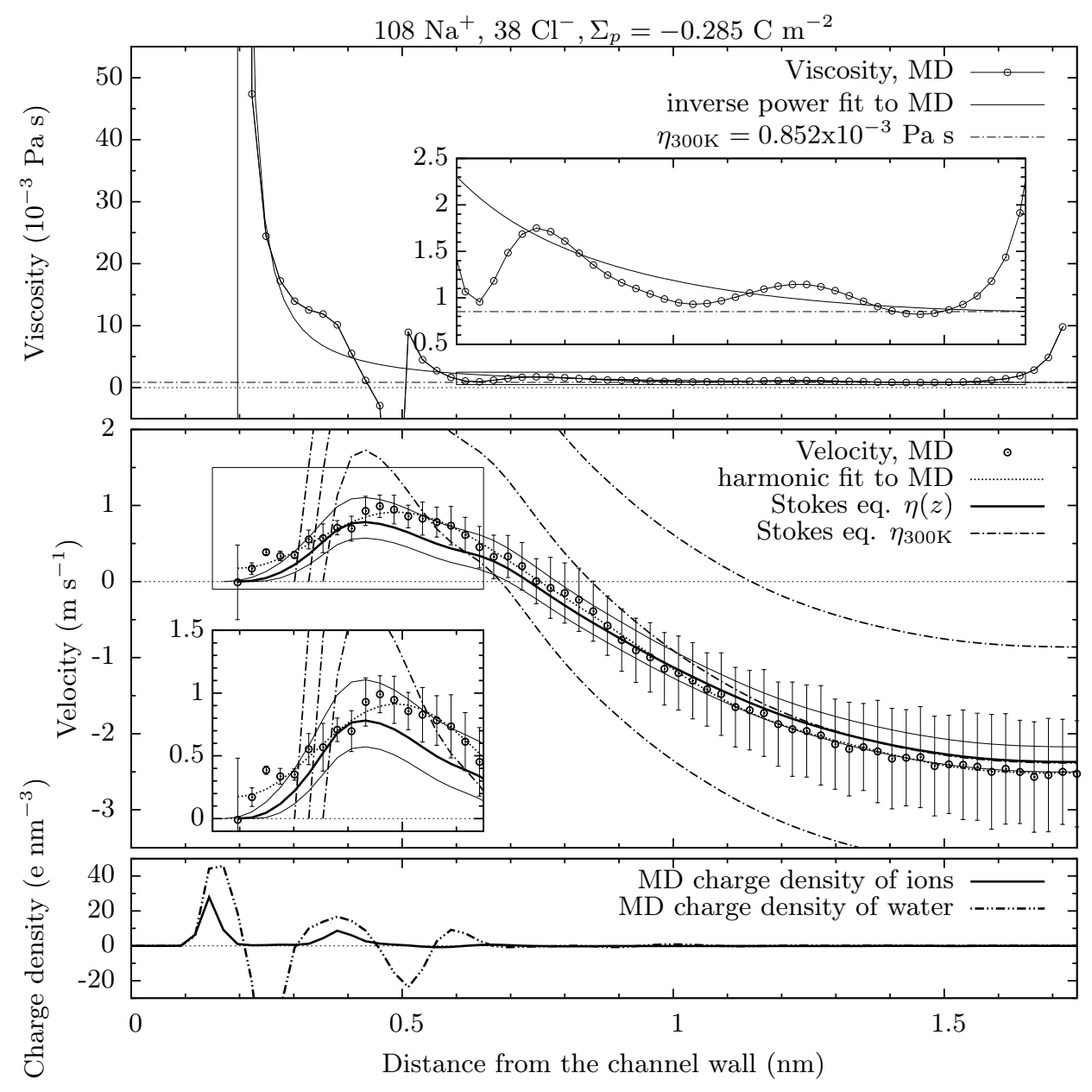




\section{Effects of spatial viscosity variation on water velocity profile at high surface charge density}

To introduce an approximate representation of the cross-channel variation of water viscosity, the viscosity estimate of the reference system from equation (6) was fit to an inverse power function

$$
\eta(z)=\left[1-\left(\frac{z}{h}\right)^{2}\right]^{-p} \eta_{\exp }
$$

where $p$ is a positive parameter and the $\eta_{\exp }$ is the experimental viscosity of water at $\mathrm{T}=300 \mathrm{~K}$. This function, shown on the top plot in Figure 9 as a solid curve, enforces $\eta(0)=\eta_{\exp }$ and $\eta(h)=\eta(-h)=\infty$. With $h=1.549 \mathrm{~nm}$ (MD distance of the furthest atom from the channel center), the least square fit results in $p=1.26$.

Solutions $u_{x}(z)$ of the Stokes equation (5) supplied with MD ionic charge densities and variable viscosity given by equation (8) are represented as solid line curves on the middle plot in Figure 9. The thick solid line curve imposed the non-slip boundary condition (BC) at the distance $0.196 \mathrm{~nm}$ from the channel wall $(h=1.549 \mathrm{~nm})$. As expected, variable viscosity curves match the MD velocity profile very well - variable viscosity was fit to satisfy the Stokes equation. Replacement of constant viscosity by a simple inverse power function leads to a significant improvement in prediction of MD velocity profile for the strongly charged surface. Although the Stokes equation was used to estimate variation of viscosity in the past (Davis \& Leighton 1987, Freund 2002, Qiao \& Aluru 2003), present authors are not aware of the past efforts to predict velocity profile using non-constant viscosity. In reference to the discussion in Zhu et al. (2005), the middle plot in Figure 9 clarifies "the extent to which hydrodynamic predictions are affected by increased viscosity in the narrow near-wall region". It manifests that the spatial viscosity variation, if present, can have a strong effect on the velocity profile of the nanochannel electro-osmotic flow.

To check the sensitivity of predicted velocity profiles on the BC location, two adjacent curves (thin solid lines) show solutions of the Stokes equation with the non-slip BC shifted one bin width $(0.026 \mathrm{~nm})$ closer to the channel wall ( $p=$ $1.22)$ and one bin width further from the channel wall $(p=1.26)$-demonstrating that velocity profile predictions using variable viscosity are less sensitive to the $\mathrm{BC}$ location than those with constant viscosity. For different surface conditions (uncharged surface) the inverse power approximation may not be appropriate, but for the surface conditions of the reference system (polar water molecules close to strongly charged surface) this approximation provides a good representation of viscosity variation in the near-wall region. It leads to an improved prediction of velocity profiles from charge density and it avoids the need for boundary condition adjustment - the location of the oxygen atom nearest to the channel wall is used.

\section{Conclusion}

The variation of a nanochannel electro-osmotic flow with surface charge density and distribution was examined. Uniformly charged fcc and diamond surface structures 
showed different quantitative, but similar qualitative behavior. At intermediate surface charge densities, intensity of the flow exhibits a maximum. The flow decreased at close-to-zero surface charge densities, and was also suppressed and even reversed at high (in absolute value) surface charge densities. A profound effect of the surface charge distribution was found. In comparison with uniform partial charge distributed on all surface atoms, the flow with discrete -1 e charge on a subset of the surface atoms was strongly suppressed and the flow reversal needed much lower surface charge density to appear. The mechanisms that lead to the surface charge dependence of a nanochannel flow were found to be adsorption and immobilization of counterions on the surface, charge redistribution, and an increase in the near-wall water viscosity at high surface charge densities. The adsorption of counterions on the surface, caused by their strong Coulomb attraction to highly charged surface atoms, led to their immobilization and to dominance of coions in the channel center. An increase in the near-wall water viscosity and its significant effects on the velocity profile of water were demonstrated using a simple inverse power approximation of the cross-channel viscosity profile. Presented results suggest that a proper representation of the surface charge density and distribution are critical parameters of the nanochannel electro-osmotic flow models.

\section{Acknowledgments}

This work was funded by the US Army Corps of Engineers through contract number W912HZ-09-C-0024. Computational resources at the MSU HPC ${ }^{2}$ center were used. Computational package LAMmPs (Plimpton 1995) was used to perform MD simulations. Permission to publish this material was granted by the Director of the Geotechnical and Structures Laboratory of the Engineer Research and Development Center, U.S. Army Corps of Engineers.

\section{References}

Abraham, F. F. (1978), 'The interfacial density profile of a Lennard-Jones fluid in contact with a (100) Lennard-Jones wall and its relationship to idealized fluid/wall systems: A Monte Carlo simulation', J. Chem. Phys. 68(8), 3713-3716. URL: http://dx.doi.org/10.1063/1.436229 6

Berendsen, H. J. C., Grigera, J. R. \& Straatsma, T. P. (1987), 'The missing term in effective pair potentials', J. Phys. Chem. 91(24), 6269-6271. URL: http://dx.doi.org/10.1021/j100308a038 3

Bonthuis, D. J., Horinek, D., Bocquet, L. \& Netz, R. R. (2009), 'Electrohydraulic power conversion in planar nanochannels', Phys. Rev. Lett. 103(14), 144503. URL: http://dx.doi.org/10.1103/PhysRevLett.103.144503 4

Cory, S. M., Liu, Y. \& Glavinović, M. I. (2007), 'Interfacial interactions of glutamate, water and ions with carbon nanopore evaluated by molecular dynamics simulations', Biochim. Biophys. Acta, Biomembr. 1768(9), 2319-2341. URL: http://dx.doi.org/10.1016/j.bbamem.2007.06.006 3

Cruz-Chu, E. R., Aksimentiev, A. \& Schulten, K. (2009), 'Ionic Current Rectification through Silica Nanopores', J. Phys. Chem. C 113(5), 1850-1862. URL: http://dx.doi.org/10.1021/jp804724p 3 
Davis, R. H. \& Leighton, D. T. (1987), 'Shear-induced transport of a particle layer along a porous wall', Chem. Eng. Science 42(2), 275-281. URL: http://dx.doi.org/10.1016/0009-2509(87)85057-1 14, 16

Dove, P. M. \& Craven, C. M. (2005), 'Surface charge density on silica in alkali and alkaline earth chloride electrolyte solutions', Geochim. Cosmochim. Acta 69(21), 4963-4970. URL: http://dx.doi.org/10.1016/j.gca.2005.05.006 4

Evans, D. J. \& Morriss, G. P. (1986), 'Shear thickening and turbulence in simple fluids', Phys. Rev. Lett. 56(20), 2172-2175.

URL: http://dx.doi.org/10.1103/PhysRevLett.56.2172/5

Freund, J. B. (2002), 'Electro-osmosis in a nanometer-scale channel studied by atomistic simulation', J. Chem. Phys. 116(5), 2194-2200. URL: http://dx.doi.org/10.1063/1.1431543, $2,12,16$

Gumbart, J., Wiener, M. C. \& Tajkhorshid, E. (2009), 'Coupling of Calcium and Substrate Binding through Loop Alignment in the Outer-Membrane Transporter BtuB', J. Mol. Biol. 393(5), 1129-1142.

URL: http://dx.doi.org/10.1016/j.jmb.2009.09.004 3

Han, B.-G., Guliaev, A. B., Walian, P. J. \& Jap, B. K. (2006), 'Water Transport in AQP0 Aquaporin: Molecular Dynamics Studies', J. Mol. Biol. 360(2), 285-296. URL: http://dx.doi.org/10.1016/j.jmb.2006.04.039 3

Hess, B., Kutzner, C., van der Spoel, D. \& Lindahl, E. (2008), 'GROMACS 4: Algorithms for Highly Efficient, Load-Balanced, and Scalable Molecular Simulation', J. Chem. Theory Comput. 4(3), 435-447.

URL: http://dx.doi.org/10.1021/ct700301q 3

Hockney, R. W. \& Eastwood, J. W. (1989), Computer Simulation Using Particles, Adam Hilger, Bristol. 3

Hoover, W. G. (1985), 'Canonical dynamics: Equilibrium phase-space distributions', Phys. Rev. A 31(3), 1695-1697. URL: http://dx.doi.org/10.1103/PhysRevA.31.1695 4

Huang, D. M., Cottin-Bizonne, C., Ybert, C. \& Bocquet, L. (2007), 'Ion-Specific Anomalous Electrokinetic Effects in Hydrophobic Nanochannels', Phys. Rev. Lett. 98(17), 177801.

URL: http://dx.doi.org/10.1103/PhysRevLett.98.177801, $2,11,12$

Hunter, R. J. (1981), Zeta potential in colloid science: principles and applications, Academic Press, London. 13

Hunter, R. J. (1987), Foundations of colloid science, 2 edn, Oxford University Press, New York. 13

Israelachvili, J. (1985), Intermolecular and surface forces, Academic press, London. 4

Jelinek, B., Felicelli, S. D., Mlakar, P. F. \& Peters, J. F. (2009), Molecular Dynamics Study of Temperature Effects on Electrokinetic Transport in Si Nanochannel, in 'Procs. ASME Int. Mech. Eng. Congr. Exp.', ASME, pp. 1511-1515.

URL: http://dx.doi.org/10.1115/IMECE2009-11690 9

Joseph, S. \& Aluru, N. R. (2006), 'Hierarchical multiscale simulation of electrokinetic transport in silica nanochannels at the point of zero charge', Langmuir 22(21), 90419051.

URL: http://dx.doi.org/10.1021/la0610147 2

Lin, Y.-S., Lin, J.-H. \& Chang, C.-C. (2010), 'Molecular Dynamics Simulations of the Rotary Motor F0 under External Electric Fields across the Membrane', Biophys. J. 98(6), 1009-1017.

URL: http://dx.doi.org/10.1016/j.bpj.2009.11.025 3 
Lorenz, C. D., Crozier, P. S., Anderson, J. A. \& Travesset, A. (2008), 'Molecular Dynamics of Ionic Transport and Electrokinetic Effects in Realistic Silica Channels', J. Phys. Chem. C 112(27), 10222-10232.

URL: http://dx.doi.org/10.1021/jp711510k 2 , 3

Lyklema, J., Rovillard, S. \& De Coninck, J. (1998), 'Electrokinetics: The properties of the stagnant layer unraveled', Langmuir 14(20), 5659-5663.

URL: http://dx.doi.org/10.1021/la980399t 6

Martínez, L., Andrade, R., Birgin, E. G. \& Martínez, J. M. (2009), 'PACKMOL: A package for building initial configurations for molecular dynamics simulations', $J$. Comput. Chem. 30(13), 2157-2164.

URL: http://dx.doi.org/10.1002/jcc.21224 4

Netz, R. R. (2003), 'Electrofriction and Dynamic Stern Layers at Planar Charged Surfaces', Phys. Rev. Lett. 91(13), 138101.

URL: http://dx.doi.org/10.1103/PhysRevLett.91.1381016

Nosé, S. (1984), 'A unified formulation of the constant temperature molecular dynamics methods', J. Chem. Phys. 81(1), 511-519.

URL: http://dx.doi.org/10.1063/1.447334 4

Patra, M. \& Karttunen, M. (2004), 'Systematic comparison of force fields for microscopic simulations of $\mathrm{NaCl}$ in aqueous solutions: Diffusion, free energy of hydration, and structural properties', J. Comput. Chem. 25(5), 678-689. URL: http://dx.doi.org/10.1002/jcc.10417 3

Plimpton, S. J. (1995), 'Fast Parallel Algorithms for Short-Range Molecular Dynamics', J. Comput. Phys. 117(1), 1-19.

URL: http://dx.doi.org/10.1006/jcph.1995.1039 17

Plimpton, S. J., Pollock, R. \& Stevens, M. (1997), Particle-Mesh Ewald and rRESPA for Parallel Molecular Dynamics Simulations, in 'Procs. 8th SIAM Conf. Par. Proc. Sci. Comput.', SIAM, pp. 8-21.

URL: http://www.siam.org/meetings/archives/pp97/cp11.htm 3

Qiao, R. \& Aluru, N. R. (2003), 'Ion concentrations and velocity profiles in nanochannel electroosmotic flows', J. Chem. Phys. 118(10), 4692-4701.

URL: http://dx.doi.org/10.1063/1.1543140 14,16

Qiao, R. \& Aluru, N. R. (2004), 'Charge inversion and flow reversal in a nanochannel electro-osmotic flow', Phys. Rev. Lett. 92(19), 198301. URL: http://dx.doi.org/10.1103/PhysRevLett.92.198301, $3,4,6$

Rapaport, D. C. (2004), The Art of Molecular Dynamics Simulation, Cambridge University Press, Cambridge, UK. 4

Rotenberg, B., Marry, V., Dufreche, J.-F., Giffaut, E. \& Turq, P. (2007), 'A multiscale approach to ion diffusion in clays: Building a two-state diffusion-reaction scheme from microscopic dynamics', J. Coll. Interf. Sci. 309(2), 289-295. URL: http://dx.doi.org/10.1016/j.jcis.2007.01.090 3

Ryckaert, J.-P., Ciccotti, G. \& Berendsen, H. J. C. (1977), 'Numerical integration of the cartesian equations of motion of a system with constraints: molecular dynamics of n-alkanes', J. Comput. Phys. 23(3), 327-341. URL: http://dx.doi.org/10.1016/0021-9991(77)90098-5 4

Schoch, R. B., van Lintel, H. \& Renaud, P. (2005), 'Effect of the surface charge on ion transport through nanoslits', Phys. Fluids 17(10), 100604. URL: http://dx.doi.org/10.1063/1.1896936 3

Sendner, C., Horinek, D., Bocquet, L. \& Netz, R. R. (2009), 'Interfacial water at hydrophobic and hydrophilic surfaces: Slip, viscosity, and diffusion', Langmuir 25(18), 10768-10781.

URL: http://dx.doi.org/10.1021/la901314b 14 
ThermExcel (2003), 'Physical characteristics of water (at the atmospheric pressure)', http://www.thermexcel.com/english/tables/eau_atm.htm. 13,14

Xia, X. \& Berkowitz, M. L. (1995), 'Electric-Field Induced Restructuring of Water at a Platinum-Water Interface: A Molecular Dynamics Computer Simulation', Phys. Rev. Lett. 74(16), 3193-3196.

URL: http://dx.doi.org/10.1103/PhysRevLett.74.31936

Yeh, I.-C. \& Berkowitz, M. L. (1999), 'Ewald summation for systems with slab geometry', J. Chem. Phys. 111(7), 3155-3162.

URL: http://dx.doi.org/10.1063/1.479595 4

Zhu, W., Singer, S. J., Zheng, Z. \& Conlisk, A. T. (2005), 'Electro-osmotic flow of a model electrolyte', Phys. Rev. E 71(4), 041501.

URL: http://dx.doi.org/10.1103/PhysRevE.71.041501 2 16 